\title{
Author Correction: Human sex reversal is caused by duplication or deletion of core enhancers upstream of SOX9
}

\author{
Brittany Croft (1) 1,2,12, Thomas Ohnesorg ${ }^{1,3,12}$, Jacqueline Hewitt ${ }^{1,4,5}$, Josephine Bowles (1) 6,7, \\ Alexander Quinn (10 7, Jacqueline Tan ${ }^{7}$, Vincent Corbin (1) ${ }^{8}$, Emanuele Pelosi ${ }^{7}$, Jocelyn van den Bergen ${ }^{1}$, \\ Rajini Sreenivasan (1,9, Ingrid Knarston 1,3, Gorjana Robevska1, Dung Chi Vu10, John Hutson 1,3,11, \\ Vincent Harley (1) ${ }^{9}$, Katie Ayers ${ }^{1,3}$, Peter Koopman \& Andrew Sinclair ${ }^{1,3}$
}

Correction to: Nature Communications https:/doi.org/10.1038/s41467-018-07784-9, published online 14 December 2018.

The original version of this Article contained an error in the spelling of the author Jacqueline Hewitt, which was incorrectly given as Jacky Hewitt. This has now been corrected in both the PDF and HTML versions of the Article.

Published online: 23 July 2019

Open Access This article is licensed under a Creative Commons Attribution 4.0 International License, which permits use, sharing, adaptation, distribution and reproduction in any medium or format, as long as you give appropriate credit to the original author(s) and the source, provide a link to the Creative Commons license, and indicate if changes were made. The images or other third party material in this article are included in the article's Creative Commons license, unless indicated otherwise in a credit line to the material. If material is not included in the article's Creative Commons license and your intended use is not permitted by statutory regulation or exceeds the permitted use, you will need to obtain permission directly from the copyright holder. To view a copy of this license, visit http://creativecommons.org/licenses/by/4.0/.
\end{abstract}

(C) The Author(s) 2019

\footnotetext{
${ }^{1}$ Murdoch Children's Research Institute, Melbourne 3052 VIC, Australia. ${ }^{2}$ Department of Molecular \& Translational Science, Monash University, Clayton 3800 VIC, Australia. ${ }^{3}$ Department of Paediatrics, The University of Melbourne, Melbourne 3010 VIC, Australia. ${ }^{4}$ School of Bioscience, University of Melbourne, Melbourne 3010 VIC, Australia. ${ }^{5}$ Department of Paediatric Urology, Monash Children's Hospital, Clayton 3168 VIC, Australia. ${ }^{6}$ School of Biomedical Sciences, University of Queensland, Brisbane 4072 QLD, Australia. ${ }^{7}$ Institute for Molecular Bioscience, The University of Queensland, Brisbane 4072 QLD, Australia. ${ }^{8}$ Bioinformatics Division, Walter \& Eliza Hall Institute of Medical Research, Melbourne 3052 VIC, Australia. ${ }^{9}$ Hudson Institute for Medical Research, Clayton 3168 VIC, Australia. ${ }^{10}$ Department of Medical Genetics, Metabolism \& Endocrinology, National Children's Hospital, Hanoi, Vietnam. ${ }^{11}$ Department of Urology, Royal Children's Hospital, Melbourne 3052 VIC, Australia. ${ }^{12}$ These authors contributed equally: Brittany Croft, Thomas Ohnesorg. Correspondence and requests for materials should be addressed to A.S. (email: Andrew.sinclair@mcri.edu.au)
} 